\title{
Lipopolysaccharide Preconditioning Reduces Neuroinflammation Against Hypoxic Ischemia and Provides Long-Term Outcome of Neuroprotection in Neonatal Rat
}

\author{
HSIANG-YIN LIN, CHAO-CHING HUANG, AND KANG-FAN CHANG \\ Institute of Basic Medical Sciences [H.-Y.L.], Institute of Clinical Medicine [C.-C.H.], and Department of Pediatrics [C.-C.H., K.-F.C.], \\ National Cheng Kung University College of Medicine, Tainan City 70428, Taiwan
}

\begin{abstract}
Hypoxic ischemia (HI) in newborns causes long-term neurologic abnormalities. Systemic lipopolysaccharide (LPS) is neuroprotective in neonatal rats when injected $24 \mathrm{~h}$ before HI. However, the effect on HI-induced neuroinflammation and the long-term outcome of LPS preconditioning in neonatal rats have not been examined. In a rat-pup HI model, compared with normal saline (NS), 0.3 $\mathrm{mg} / \mathrm{kg}$ of LPS injected $24 \mathrm{~h}$ before HI greatly increased microglial cell and macrophage activation and up-regulated TNF-alpha and inducible NOS expression 12-h postinjection and resulted in high mortality during HI. In contrast, $0.05 \mathrm{mg} / \mathrm{kg}$ of LPS elicited very little microglia and macrophage activation and TNF-alpha and inducible NOS expression and resulted in low mortality. Given $24 \mathrm{~h}$ before HI, low-dose $(0.05 \mathrm{mg} / \mathrm{kg})$ LPS greatly reduced microglia and macrophage activation, TNF-alpha expression, and reactive oxygen species production 24-h post-HI compared with NS-treated rats. Rats in the low-dose LPS group also showed significantly better learning and memory and less brain damage in adulthood. Learning and memory performance among the LPS-HI, LPS, and NS groups was not significantly different. We conclude that low-dose LPS preconditioning in neonatal rats greatly reduces HI-induced neuroinflammation and provides long-term neuroprotection against behavioral and pathologic abnormalities. (Pediatr Res 66: 254-259, 2009)
\end{abstract}

$\mathrm{T}$ wenty percent of newborns with hypoxic ischemia (HI) die and an additional $25 \%$ have long-term neurodevelopmental handicaps (1). Although hypothermia has been used to treat newborns with HI encephalopathy, there is no effective pharmacological therapy. Sublethal lipopolysaccharide (LPS) stimulation, preconditioning, protects against subsequent lethal injury. Elucidating the neuroprotective mechanisms of LPS preconditioning may offer potential therapies against HI brain injury $(2,3)$.

Systemic LPS preconditioning (0.5 to $0.9 \mathrm{mg} / \mathrm{kg}$ ) 48 to $72 \mathrm{~h}$ before ischemic insults protected adult rats against cerebral ischemia $(4,5)$. LPS $(0.3$ or $1 \mathrm{mg} / \mathrm{kg})$ sensitized neonatal Wistar rats and worsened HI brain injury when given 4,6 , or $72 \mathrm{~h}$ before HI (6). In contrast, $0.3 \mathrm{mg} / \mathrm{kg}$ of LPS $24 \mathrm{~h}$ before HI protected them $(7,8)$. There are several neuroprotective preconditioning models for the neonatal brain $(7,9-13)$, but

Received January 26, 2009; accepted April 10, 2009.

Correspondence: Chao-Ching Huang, MD, Department of Pediatrics, National Cheng Kung University Hospital, 138 Sheng-Li Road, Tainan City 70428, Taiwan; e-mail: huangped@mail.ncku.edu.tw

Supported by grants from the Taiwan National Health Research Institute (NHRIEX94, 95-9414NI), the National Science Counsel (95-2314-B-006-012; 96-2314B006-004), and the Center for Gene Regulation and Signal Transduction Research, National Cheng Kung University. there are only few evaluations of long-term outcomes of LPS preconditioning at behavioral and pathologic levels.

Inflammatory responses are important for developing neonatal HI brain injury (1). Up-regulated reactive oxygen species (ROS) production is also critical in inducing HI damage in the neonatal brain $(1,14)$. One study $(15)$ of adult rats showed that low-dose $(0.05 \mathrm{mg} / \mathrm{kg})$ LPS pretreatment $24 \mathrm{~h}$ before transient middle-cerebral-artery occlusion decreased ischemic infarct size despite increases of inflammatory cells in the ischemic hemisphere. In contrast, another study (16) suggested that LPS $(0.2 \mathrm{mg} / \mathrm{kg})$ preconditioning $48 \mathrm{~h}$ before transient middlecerebral-artery occlusion suppressed neuroinflammatory responses. Although up-regulating endogenous corticosterone was important in LPS-induced HI tolerance in neonatal rats (17), it is not clear whether LPS preconditioning diminished HI-induced inflammation and ROS production in the neonatal brain. Therefore, we tested the following hypotheses: 1) the long-term outcome of LPS preconditioning against HI in neonatal rats can be established at behavioral and pathologic levels; and 2) the mechanism of LPS preconditioning involves suppressing HI-induced neuroinflammation.

\section{MATERIALS AND METHODS}

This study was approved by the Animal Care Committee at National Cheng Kung University. Ten to 12 Sprague-Dawley female rat pups per dam were housed with a 12/12-h light/dark schedule. The pups were housed with their dams until weaning on postpartum day 21 (P21) and cared for in accordance with the National Institutes of Health guidelines.

LPS pretreatment in rat pups. We injected rat pups with $0.05 \mathrm{mg} / \mathrm{kg}$ (LPS0.05) or $0.3 \mathrm{mg} / \mathrm{kg}$ of LPS (LPS0.3) (i.p.) (Escherichia coli 0111:B4; Sigma Chemical Co.-Aldrich, St. Louis, MO) or pyrogen-free normal saline (NS) 3 or $24 \mathrm{~h}$ before HI on P7. Because of the high HI-mortality in rat pups pretreated with LPS0.3, we reduced the dose to LPS0.05 mg/kg for preconditioning. Ninety-seven rat pups were sorted into four groups: NS ( $n=24$, NS injected on P6 without HI), LPS ( $n=20$, LPS 0.05 on P6 without HI), NS-HI $(n=23, \mathrm{NS}$ injected $24 \mathrm{~h}$ before HI), and LPS-HI $(n=30$, LPSO.05 $24 \mathrm{~h}$ before HI). To avoid LPS-induced body temperature changes during preconditioning, the rat pups were returned to their dams after a LPS or NS injection and housed in an incubator to maintain body temperature. Mean body temperature was comparable between the LPS0.05 $\left(34.8 \pm 0.5^{\circ} \mathrm{C}\right)$ and NS groups $\left(34.8 \pm 0.6^{\circ} \mathrm{C}\right)$ before HI. The right common carotid artery was permanently ligated in P7 pups under $2.5 \%$ halothane anesthesia. After surgery, the pups were returned to the incubator for a 1-h recovery. They were then placed in airtight $500-\mathrm{mL}$ containers partially submerged in a $37^{\circ} \mathrm{C}$ water

\footnotetext{
Abbreviations: HI, hypoxic ischemia; IHC, immunohistochemistry; iNOS, inducible NOS; LPS, lipopolysaccharide; NS, normal saline; ROS, reactive oxygen species
} 
bath, and humidified $8 \%$ oxygen was kept at a flow rate of $3 \mathrm{~L} / \mathrm{min}$ for $2 \mathrm{~h}$. After hypoxia, the rat pups were returned to their dams.

Immunohistochemistry. The immunohistochemistry (IHC) for microglial cell activation and TNF-alpha expression was done at $12 \mathrm{~h}$ after LPS or NS injection and at $24 \mathrm{~h}$ after HI. After the brains had been removed and postfixed in $4 \%$ paraformaldehyde overnight, they were dehydrated using $30 \%(\mathrm{wt} / \mathrm{vol})$ sucrose in PBS for $2 \mathrm{~d}$ and then coronally sectioned (14- $\mu \mathrm{m}$ thick) from the genu of the corpus callosum to the end of the dorsal hippocampus. The specific primary antibodies included anti-rat TNF-alpha antibody (1:100) (Cat. \# BMS175; Bender MedSystems, Vienna, Austria) and anti-rat microglia and macrophage antibody (CD-11b; 1:100) (Cat. \# MCA275R; AbD Serotec, Oxford, UK). Biotinylated goat anti-mouse, anti-goat, or anti-rabbit IgG was used (all 1:200). Biotin signals were detected using $0.5 \mathrm{mg} / \mathrm{mL}$ 3'3'-diaminobenzidine (DAB) $/ 0.003 \% \mathrm{H}_{2} \mathrm{O}_{2}$ as a substrate. Results were recorded using a microscope (E400; Nikon Instech, Kawasaki, Japan).

$\mathrm{CD}-11 \mathrm{~b}$ and TNF-alpha expression was quantitatively analyzed in the cross-sectional areas of the cortex and white matter in two reference planes corresponding to plates 31 and 39 in a rat brain atlas (18). The number of CD-11b-(+) cells and the integrated OD (IOD) of TNF-alpha signals were analyzed, using imaging software (ImagePro Plus 6.0; Media Cybernetics, Bethesda, MD) at $400 \times$ magnification per visual field (one visual field $=$ $0.096 \mathrm{~mm}^{2}$ ). Three visual fields within a cross-sectional area in the cortex and white matter were analyzed and averaged.

Immunoblotting. The cortex was homogenized (12), and samples were resolved using $10 \%$ SDS-PAGE and then blotted electrophoretically to polyvinylidene fluoride membranes. The membranes were incubated with primary antibodies: anti-rat inducible NOS (iNOS) (1:1000) (Cat. \# sc651; Santa Cruz, CA) or anti-actin antibody (1:1000) (Cat. \# MAB 1501R; Chemicon, Temecula, CA). Immunoreactivity was detected using horseradish peroxidaseconjugated anti-rabbit or anti-mouse IgG antibody (1:10,000) (Cat. \# 401315 and 101215, respectively; Calbiochem, Darmstadt, Germany) and visualized using enhanced chemiluminescence (Pierce Biotechnology, Rockford, IL). VisionWorks LS (Ultra-Violet Products Ltd., Cambridge, UK) analysis software was used for densitometry, and iNOS levels were assessed after they were normalized to actin expression.

ROS production. ROS production was detected with a chemiluminescence detector (19). The cortex of each group's brain was weighed and homogenized, and then its chemiluminescence was measured (Luminex 200; Luminex Corp., Austin, TX). After determining the background level for $100 \mathrm{~s}$, we injected into the sample $1.0 \mathrm{~mL}$ of $0.1 \mathrm{mM}$ luminal (5-amino-2,3-dihydro1,4-phthalazinedione) (Sigma Chemical Co.-Aldrich) in NS. The chemiluminescence was monitored continuously for an additional $500 \mathrm{~s}$. Total chemiluminescence was calculated by integrating the area under the curve and subtracting it from the background level. The ROS assay was expressed as chemiluminescence counts $/ 10 \mathrm{~s} / \mathrm{mg}$ of the brain tissue.

Long-term outcome measures. Rats underwent the water maze, as previously described $(12,20)$. In brief, the pool was divided into four quadrants. An $8 \mathrm{~cm} \times 8 \mathrm{~cm}$ Plexiglas platform, onto which the rats could escape from the water, was placed $1 \mathrm{~cm}$ below the water surface in the center of one quadrant. The escape distances and latencies, and the swimming speeds and patterns of the rats were monitored using a camera above the pool and connected to a computer program (Noldus Ethovision, Wageningen, The Netherlands). The task was divided into five phases.

Phase I. Acquisition (days 1-2) consisted of $2 \mathrm{~d}$ of training with two sessions per day. Each session consisted of four trials with four different starting positions. After swimming from each of the starting positions and mounting the submerged platform, the rats were allowed to remain there for $60 \mathrm{~s}$. If a rat did not find the platform within $120 \mathrm{~s}$, it was directly placed on the platform and allowed a 60-s rest period.

Phase II. Probe test 1 (day 3), the platform was removed from the pool, and the rats were placed in the pool in the quadrant opposite the previous platform position. The rats were allowed $60 \mathrm{~s}$ of free swimming. The percentage of time spent in the target quadrant was calculated.

Phase III. Reversal training (days 4-5) was done by placing the platform at a new position in the quadrant opposite the one used in Phase I. Reversal training used the procedure described in Phase I.

Phase IV. Probe test 2 (Day 6) used the procedure described in Phase II. The percentage of time spent in the new target quadrant was calculated.

Phase V. Visible platform test (Day 6) occurred $2 \mathrm{~h}$ after the probe test. The rats were released from a specific location and required to locate a green escape platform $2 \mathrm{~cm}$ above the water surface in a position not used during the former sessions. If the rat did not reach the platform within $120 \mathrm{~s}$, it was recorded as $120 \mathrm{~s}$. Swimming speed was measured across the training sections in the acquisition and reversal phases.

Brain damage calculated using hemispheric weight reduction on P42. The brains were sectioned at the midline. The left and right hemispheres were weighed. The percentage of hemispheric weight reduction, measured as [(left hemisphere weight - right hemisphere weight)/left hemisphere weight], was used as the measure of cerebral injury (12). After the hemispheres had been weighed, representative brains from each group were postfixed with $4 \%$ paraformaldehyde and then alcohol dehydrated. After they had been infiltrated with xylene, both hemispheres from each rat were embedded in paraffin in the same cassette, coronally sectioned (10- $\mu \mathrm{m}$ thick) at the mid-hippocampus, and stained with cresyl violet.

Statistical analysis. Statistical significance $(p<0.05)$ was determined using one-way ANOVA or $t$ test. MANOVA was used to compare escape time over the learning phase of the water maze. Tukey's method was used for post hoc comparisons in one-way ANOVA, and the Bonferroni method in MANOVA. Continuous data are means \pm SEM.

\section{RESULTS}

Effects of LPS on microglia and TNF-alpha. CD-11b IHC showed that compared with NS, there were few activated microglia and macrophage in the cerebral cortex $12 \mathrm{~h}$ after LPS injection (Fig. 1A). LPS0.3 significantly increased the number of CD-11b- $(+)$ cells in white matter than LPS0.05 $(p<0.05)$ and NS $(p<0.01)$ (Fig. 1B). The difference between LPS0.05 and NS groups was not significant.

Twelve hours after LPS injection, TNF-alpha expression in the cerebral cortex (Fig. 1C) and white matter (Fig. 1D) was significantly higher in the LPSO 3 group than in the LPS0.05 (both $p<0.01$ ) and NS groups (cortex, $p<0.05$; white matter, $p<0.01)$. The differences between LPS0.05 and NS groups were not significant.

Effects of LPS on iNOS expression. iNOS expression was significantly higher in the LPS0.3 group than in the NS group $3(p<0.05), 12(p<0.05)$, and $24 \mathrm{~h}(p<0.001)$ after LPS injection, and significantly higher in the LPS0.3 group than in the LPS0.05 group $12(p<0.05)$ and $24 \mathrm{~h}(p<0.01)$ postinjection. The iNOS levels were not significantly different between the LPS0.05 and NS groups (Fig. 2).

Effects of LPS on HI-mortality. We then pretreated P7 rat pups with LPS0.05 or LPS0.3 mg/kg or NS $3 \mathrm{~h}$ before HI. Both LPS groups had higher HI mortality rates (LPS0.3: 20/20, 100\%; LPS0.05: $17 / 24,70.8 \%$ ) than NS group (5/20, 25\%). These findings suggested that LPS $3 \mathrm{~h}$ before HI had sensitized the rat pups and worsened their HI-mortality. We next examined the effect of LPS $24 \mathrm{~h}$ before HI on HI-mortality. Mortality was much lower in the LPS0.05 group $(2 / 81,2.5 \%)$ than in the NS group $(18 / 92,19.6 \%)$ but much higher in the LPSO 3 group $(13 / 18,72.2 \%)$ than in the other two groups. This suggested that LPSO. 3 injected $24 \mathrm{~h}$ before HI sensitized rat pups but that LPS0.05 induced tolerance against HI.

Neuroprotective mechanisms of LPS preconditioning after HI. Twenty-four hours post-HI, the numbers of CD-11b$(+)$ microglia in the ipsilateral cerebral cortex (Fig. $3 A$ ) and white matter (Fig. 3B) were significantly (both $p<0.001$ ) increased in NS-HI than in NS group but significantly (both $p<0.01)$ lower in LPS-HI group than in NS-HI group. Differences between the NS and LPS-HI groups were not significant. TNF-alpha expression in the cerebral cortex (Fig. $3 C$ ) and white matter (Fig. 3D) $24 \mathrm{~h}$ post-HI was also significantly (both $p<0.001$ ) higher in NS-HI than in NS group but significantly (cortex, $p<0.01$; white matter, $p<0.05$ ) lower in LPS-HI than in NS-HI group. The LPS-HI group had significantly $(p<0.05)$ higher TNF-alpha levels than the NS group in the white matter but not in the cerebral cortex. 

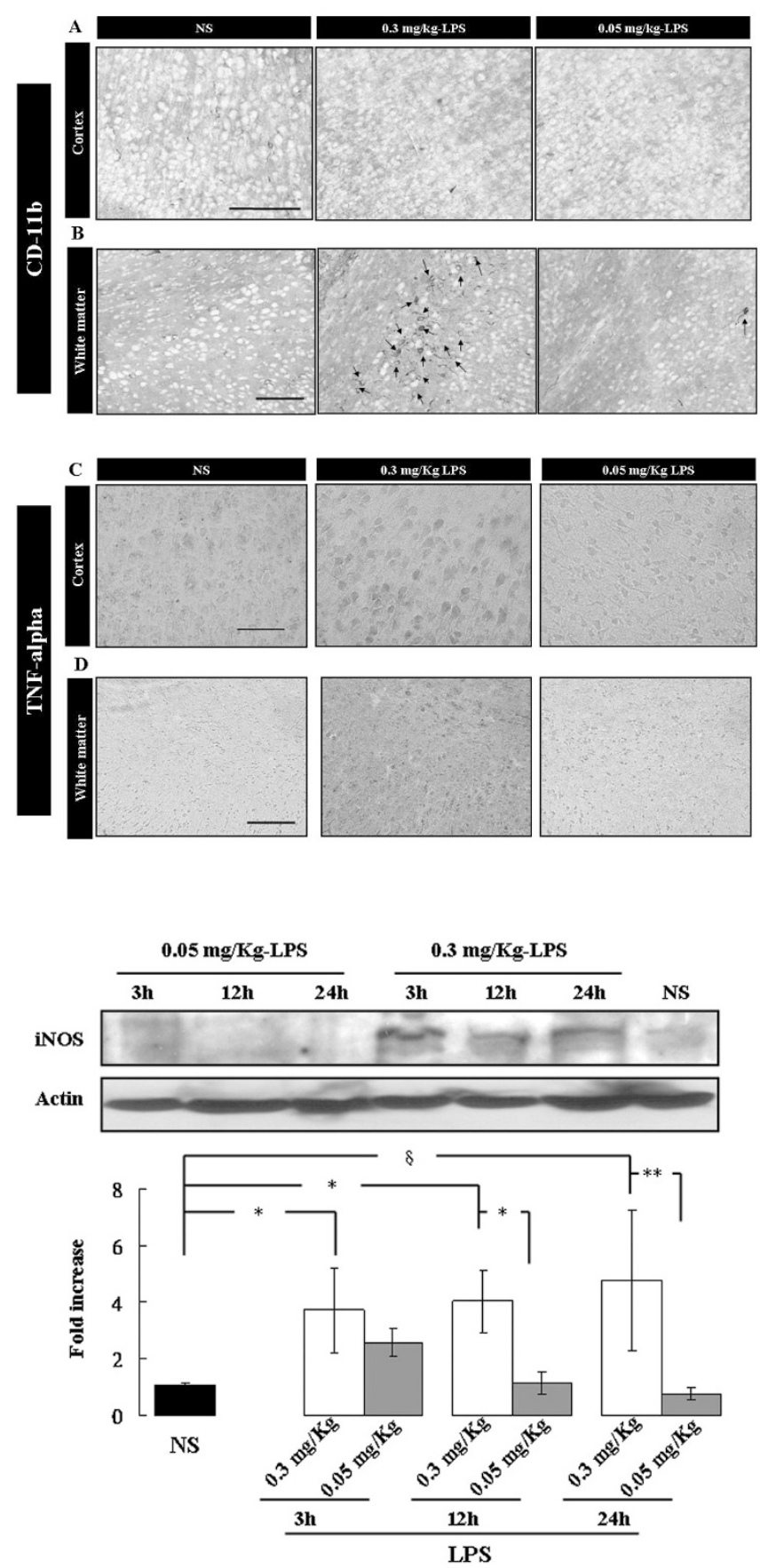

Figure 2. Immunoblotting of iNOS expression. The LPS0.3 group had significantly up-regulated iNOS at 3,12, and $24 \mathrm{~h}$ after LPS compared with the NS group and also at 12 and $24 \mathrm{~h}$ after LPS compared with the LPS0.05 group. Data are from three rats at each time point in each group. Values are means \pm SEM. $* p<0.05, * * p<0.01$, and $\S p<0.001$.

Chemiluminescence showed that, compared with NS, LPS significantly increased ROS production $(p<0.001)$ 24-h postinjection. Twenty-four hours post-HI, ROS production was significantly $(p<0.001)$ higher in NS-HI groups than in NS group (Fig. 4) but significantly $(p<0.001)$ lower in LPS-HI group than in NS-HI group.

Water maze on P35. During the learning and reversallearning phases, the four groups all learned to find the submerged platform; however, there were significant differences
Figure 1. Immunohistochemistry for CD-11b and TNF-alpha performed $12 \mathrm{~h}$ after LPS injections. LPS activated few microglias in the cerebral cortex (A) 12-h postinjection; however, there were significantly more CD-11b $(+)$ cells (arrows) in the white matter $(B)$ of rats treated with LPS0.3 than those treated with LPS0.05 or NS. TNFalpha levels were significantly higher in the cerebral cortex $(C)$ and white matter $(D)$ in the LPSO.3 group than in the other two groups (IOD, integrated OD). Differences in TNFalpha levels between the LPS0.05 and NS groups were not significant. Scale bar $=50 \mu \mathrm{m}$ in $(A, B, C)$ and $100 \mu \mathrm{m}$ in $(D)$. Data are from three rats in each group. Values are means \pm SEM. $* p<0.05$, and $* * p<0.01$

between them $(p<0.001)$. Post hoc multiple analyses showed that the LPS-HI group spent significantly less time than the NS-HI group finding the submerged platform in the learning and reversal-learning phases (both $p<0.001$ ) (Fig. 5A). There were no significant differences among LPS-HI, LPS, and NS groups. In the memory phase, there were significant differences (all $p<0.001$ ) in probe-test 1 and 2 results after the platform had been removed (Fig. 5B). Post hoc analysis showed that the LPS-HI group spent significantly more time than the NS-HI group in the target quadrant (probe test $1, p<$ 0.05 ; probe test $2, p<0.001$ ) but differences among the LPS-HI, LPS, and NS groups were not significant. Nor were there significant differences in swimming speed among the groups (Fig. $5 \mathrm{C}$ ). In the visual motor test, the NS-HI group did poorly compared with the NS $(p<0.001)$, LPS $(p<0.001)$, and LPS-HI groups $(p<0.05)$ (Fig. $5 D)$ ). Test results were not significantly different among the LPS, NS, and LPS-HI groups.

Pathology on P42. Hemispheric weight reduction analysis showed that the NS-HI group was significantly (all $p<0.001$ ) more brain damaged than the LPS-HI, LPS, and NS groups (Fig. 6). There were no significant differences among the LPS-HI, LPS, and NS groups.

\section{DISCUSSION}

Systemic LPS can increase or decrease the vulnerability to $\mathrm{HI}$ in neonatal rats. The cerebral proinflammatory cytokines, such as TNF-alpha and ROS, induced by LPS exacerbate brain damage or contribute to the induction of ischemia tolerance in adult rodents $(4,5)$. We used a lower dose of LPS than normally is used in septic shock experiments, and outcomes were different. We showed that LPSO.3 induced prominent 

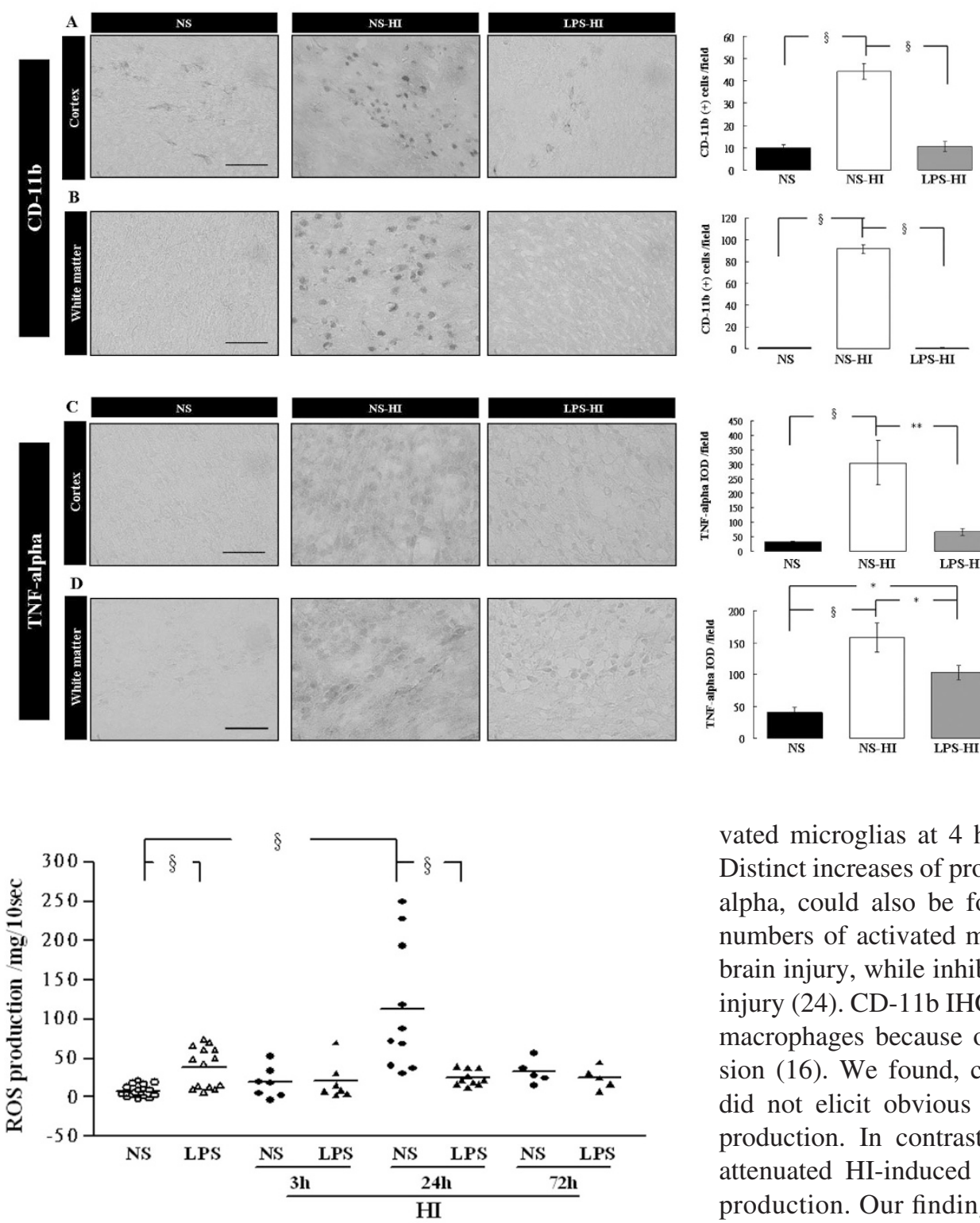

Figure 4. Chemiluminescence showed that compared with NS group, ROS production was significantly higher in LPS group 24-h postinjection. The LPS-HI group had significantly lower ROS production $24 \mathrm{~h}$ post-HI compared with the NS-HI. Values are means \pm SEM. $\S p<0.001$.

neuroinflammation, activated microglias, up-regulated TNFalpha and iNOS, and caused high mortality when given $24 \mathrm{~h}$ before HI. In contrast, the lower dose of LPSO.05 elicited significantly less neuroinflammation and caused significantly less mortality when given $24 \mathrm{~h}$ before HI. In addition, lowdose LPS preconditioning greatly reduced HI-induced microglial activation and TNF-alpha and ROS production and provided long-term neuroprotection at the behavioral and pathologic levels.

Microglia cells are critically involved with both the innate and adaptive immune systems. Activated microglias are the hallmark of HI-induced neuroinflammation and exacerbate injury in ischemic areas because of their participation in inflammatory responses (21). One of the earliest events to occur after neonatal $\mathrm{HI}$ is the appearance of abundant numbers of acti-

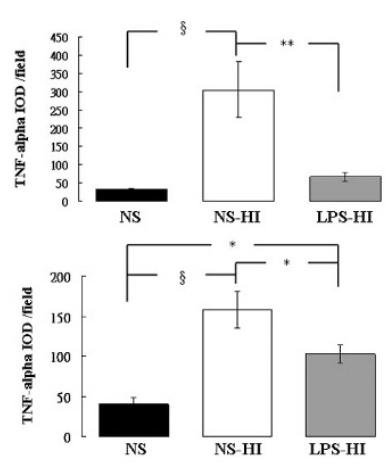
HI insult.
Figure 3. Immunohistochemistry for CD-11b and TNF-alpha performed $24 \mathrm{~h}$ after HI. The LPS-HI group had significantly reduced $\mathrm{CD}$ $11 \mathrm{~b}-(+)$ cells in the ipsilateral cortex $(A)$ and white matter $(B)$ than the NS-HI group. TNF-alpha level was also significantly decreased in the cerebral cortex $(C)$ and white matter $(D)$ in the LPS-HI group than in the NS-HI group. Data are from three rats in each group. Values are means \pm SEM. Scale bar $=50 \mu \mathrm{m}$ in $(A-D) . * p<0.05, * * p<0.01$, and $\S p<0.001$

vated microglias at $4 \mathrm{~h}$ and peaked at 2-4 d after HI (22). Distinct increases of proinflammatory cytokines, such as TNFalpha, could also be found $0-24 \mathrm{~h}$ post-HI (23). Increased numbers of activated microglias after $\mathrm{HI}$ are associated with brain injury, while inhibiting microglial activation reduces HI injury (24). CD-11b IHC staining detects activated microglias/ macrophages because of their high level of CD-11b expression (16). We found, compared with NS, LPS0.05 injection did not elicit obvious microglial activation and TNF-alpha production. In contrast, LPS0.05 preconditioning markedly attenuated HI-induced microglial activation and TNF-alpha production. Our findings are consistent with a recent report in adult mice that LPS preconditioning prevented inflammatory response in the brain during ischemia (16). Thus, low-dose LPS may induce a reprogramming process in the microglia that alters their responsiveness to a subsequent

ROS is critical in the injurious cascades of the immature brain as the levels of free radicals increase after HI $(1,14)$, and a number of ROS scavengers are neuroprotective. Oxidative injury due to excess mitochondrial production of ROS has been implicated in a number of conditions, including ischemia/reperfusion injury (25). ROS are produced by several cell types, such as microglias, neurons, and endothelial cells. The neonatal brain is particularly susceptible to oxidative stress injury because of its underdeveloped scavenging systems $(1,25)$. Electron spin resonance (ESR) detected a significant release of ROS in the neostriatum of asphyxiated newborn rats 5 min after reoxygenation (14). By chemiluminescence, we found that ROS production was significantly higher 24-h post-HI, which might be associated with the maximal impairment of mitochondrial respiration observed 24-h post-HI in neonatal rats (25). Low-dose LPS precondition- 

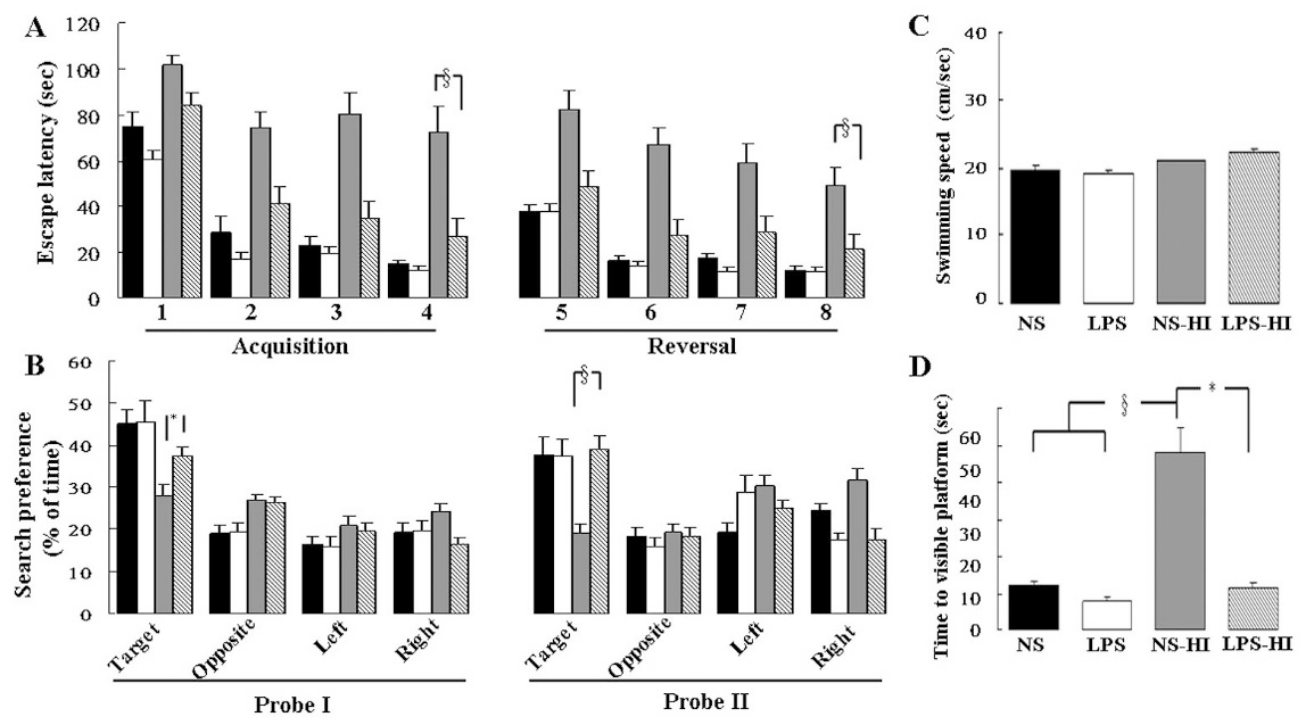

Figure 5. Low-dose LPS preconditioning provided long-term neuroprotection at the behavioral level (water-maze test). (A) The LPS-HI group spent significantly less time than the NS-HI group finding the submerged platform in the learning and reversal-learning phases. (B) The LPS-HI group also spent significantly more time in the target quadrant than did the NS-HI group in probe tests 1 and 2. Differences in the learning and memory performances among the LPS-HI, LPS, and NS groups were not significant. $(C)$ There were no significant differences in swimming speeds among the four groups. $(D)$ The visual motor test performance of NS-HI group was poorer than those of the LPS-HI, NS, and LPS groups. $\square$ : NS group, $n=24$; $\square$ : LPS group, $n=20$; $\square$ : NS-HI, $n=$ 23; $\mathbb{Q}$ : LPS-HI, $n=30$. Values are means \pm SEM. ${ }^{*} p<0.05$ and $\S p<0.001$ compared with the NS-HI group.

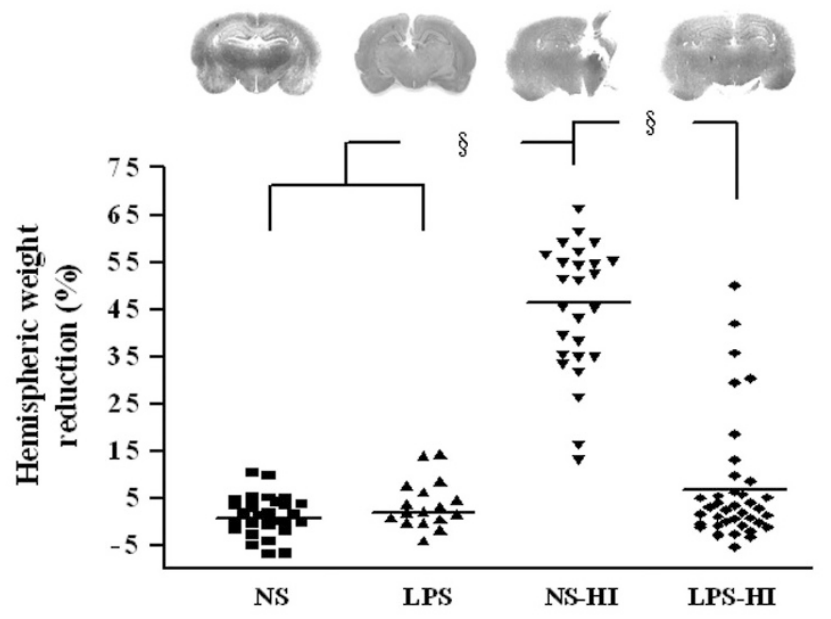

Figure 6. LPS-preconditioned showed long-term neuroprotection at the pathologic level. The upper panel shows the representative cresyl-violetstained brain sections from each group. Hemispheric weight reduction measurement showed that the NS-HI group had significantly more brain damage than did the LPS-HI, LPS, and NS groups. Values are means \pm SEM. $\S p<$ 0.001 compared with the NS-HI group.

ing greatly reduced HI-induced ROS production, which suggests that potential protective effect of low-dose LPS preconditioning at the mitochondrial level.

Long-term outcome evaluation of preconditioning in neonatal $\mathrm{HI}$ at both pathologic and behavioral levels have been reported for hypoxic (11), ligation (12), and ischemic (13) preconditioning models. Sparing neurons from pathologic damage may not necessarily translate into sparing them from dysfunction. Therefore, both pathologic and behavioral outcome assessments are important for evaluating the efficacy of potential treatments that may mitigate the consequences of brain insult (20). This study presents the first evidence showing long-term outcomes, at both the behavioral and pathologic levels, of LPS preconditioning against $\mathrm{HI}$ in neonatal rats.

One major concern of LPS preconditioning is that the LPS injection itself during neonatal period may have long-term adverse effects on behavior: a brief systemic LPS during critical periods of development may cause long-lasting cerebral vulnerability in adulthood $(7,26,27)$. Rat pups treated with LPS on P14 were significantly more susceptible to lithium-pilocarpineinduced seizures and increased hippocampal neuron degeneration after limbic seizures in adulthood. Increases in seizure susceptibility occurred only when LPS $(0.025,0.1$, or $0.25 \mathrm{mg} / \mathrm{kg})$ was given on P7 and P14, a critical postnatal period, but not on P1 or P20 (27). LPS-treated (0.1 mg/kg) P5 rat pups showed behavioral deficits in a water-maze probe trial but not in the acquisition phase at adulthood (28). In contrast, another study (26) reported that injection of $E$. coli on P4 was not associated with memory impairments in adult rats unless they were then reexposed to a second immune challenge. We showed that LPS0.05 used for preconditioning induction on P6 rat pups did not cause learning or memory impairment in adulthood.

Recent studies $(29,30)$ have suggested that gender difference may influence ischemic neuron death and the effect of neuroprotective therapy. Even though the neuroprotective effect of LPS preconditioning has been demonstrated in adult and neonatal rats (4), most of these studies have been focused on males. We have very limited information on the neuroprotective effect of LPS preconditioning in neonatal females. Therefore, we specifically examined whether the long-term outcome of neuroprotective LPS preconditioning could be established in female rat pups. Different serotypes of LPS may induce variable proinflammatory and thermoregulatory responses in adult rats. LPS is composed of lipid A, a core 
polysaccharide and peripheral $\mathrm{O}$-antigenic chains, and it has been suggested that structural differences in LPS serotypes, such as a variable content of lipid A in different LPS serotypes, produce different inflammatory responses $(31,32)$. Inflammatory cytokine responses after different LPS treatment may be stimulant dependent. The LPS serotype used in our study (E. coli O111:B4) is different from those used in used in other preconditioning studies: E. coli O55:B5 $(7,33)$ and 026:B6 (17). Our data in Sprague-Dawley rat pups showed that LPS0.3 serotype 0111:B4 injected $24 \mathrm{~h}$ before HI sensitized the rat pups and caused high HI-mortality. In contrast, LPS0.05 injected $24 \mathrm{~h}$ before $\mathrm{HI}$ induced tolerance against $\mathrm{HI}$. Studies on neonatal Wistar rats $(7,17)$ showed that higher doses of systemic LPS (serotype 026:B6, 0.3-1 mg/kg) were neuroprotective when injected $24 \mathrm{~h}$ before $\mathrm{HI}$, despite some early inflammatory molecule responses. Therefore, we suggest that the O111:B4 serotype at $0.3 \mathrm{mg} / \mathrm{kg}$ induces more potent proinflammatory responses than the O55:B5 serotype at 0.3 $\mathrm{mg} / \mathrm{kg}$ and the 026:B6 serotype at $1 \mathrm{mg} / \mathrm{kg}$. Taking all these findings together, we hypothesize that, in addition to the time interval between LPS injection and HI, the differences in animal species, LPS doses, and, more important, LPS serotypes, affects the outcome of LPS preconditioning.

Acquiring tolerance to $\mathrm{HI}$ through pharmacological preconditioning has clinical implications for perinatal medicine. Establishing the long-term outcome of LPS preconditioning in the neonatal brain, and elucidating its underlying prosurvival mechanisms, may help translational researchers discover new treatments for neonatal HI encephalopathy.

Acknowledgments. We thank Chien-Jung Ho for her skillful technical assistance with animal preparations and Mr. Bill Franke for English editing.

\section{REFERENCES}

1. Ferriero DM 2004 Neonatal brain injury. N Engl J Med 351:1985-1995

2. Chang YC, Huang CC 2006 Perinatal brain injury and regulation of transcription. Curr Opin Neurol 19:141-147

3. Gidday JM 2006 Cerebral preconditioning and ischaemic tolerance. Nat Rev Neurosci 7:437-448

4. Tasaki K, Ruetzler CA, Ohtsuki T, Martin D, Nawashiro H, Hallenbeck JM 1997 Lipopolysaccharide pre-treatment induces resistance against subsequent focal cerebral ischemic damage in spontaneously hypertensive rats. Brain Res 748:267-270

5. Rosenzweig HL, Minami M, Lessov NS, Coste SC, Stevens SL, Henshall DC, Meller R, Simon RP, Stenzel-Poore MP 2007 Endotoxin preconditioning protects against the cytotoxic effects of TNFalpha after stroke: a novel role for TNFalpha in LPS-ischemic tolerance. J Cereb Blood Flow Metab 27:1663-1674

6. Eklind S, Mallard C, Leverin AL, Gilland E, Blomgren K, Mattsby-Baltzer I, Hagberg H 2001 Bacterial endotoxin sensitizes the immature brain to hypoxicischaemic injury. Eur J Neurosci 13:1101-1106

7. Eklind S, Mallard C, Arvidsson P, Hagberg H 2005 Lipopolysaccharide induces both a primary and a secondary phase of sensitization in the developing rat brain. Pediatr Res 58:112-116
8. Mallard C, Hagberg H 2007 Inflammation-induced preconditioning in the immature brain. Semin Fetal Neonatal Med 12:280-286

9. Wada T, Kondoh T, Tamaki N 1999 Ischemic "cross" tolerance in hypoxic ischemia of immature rat brain. Brain Res 847:299-307

10. Gidday JM, Fitzgibbons JC, Shah AR, Park TS 1994 Neuroprotection from ischemic brain injury by hypoxic preconditioning in the neonatal rat. Neurosci Lett 168:221224

11. Gustavsson M, Anderson MF, Mallard C, Hagberg H 2005 Hypoxic preconditioning confers long-term reduction of brain injury and improvement of neurological ability in immature rats. Pediatr Res 57:305-309

12. Lee HT, Chang YC, Wang LY, Wang ST, Huang CC, Ho CJ 2004 cAMP response element-binding protein activation in ligation preconditioning in neonatal brain. Ann Neurol 56:611-623

13. Lin WY, Chang YC, Lee HT, Huang CC 2009 CREB activation in the rapid, intermediate, and delayed ischemic preconditioning against hypoxic-ischemia in neonatal rat. J Neurochem 108:847-859

14. Capani F, Loidl CF, Aguirre F, Piehl L, Facorro G, Hager A, De Paoli T, Farach H, Pecci-Saavedra J 2001 Changes in reactive oxygen species (ROS) production in rat brain during global perinatal asphyxia: an ESR study. Brain Res 914:204-207

15. Ahmed SH, He YY, Nassief A, Xu J, Xu XM, Hsu CY, Faraci FM 2000 Effects of lipopolysaccharide priming on acute ischemic brain injury. Stroke 31:193-199

16. Rosenzweig HL, Lessov NS, Henshall DC, Minami M, Simon RP, Stenzel-Poore MP 2004 Endotoxin preconditioning prevents cellular inflammatory response during ischemic neuroprotection in mice. Stroke 35:2576-2581

17. Ikeda T, Yang L, Ikenoue T, Mallard C, Hagberg H 2006 Endotoxin-induced hypoxic-ischemic tolerance is mediated by up-regulation of corticosterone in neonatal rat. Pediatr Res 59:56-60

18. Paxinos G, Watson C 1986 The Rat Brain in Stereotaxic Coordinates. 2nd ed Academic Press, San Diego

19. McGowan JE, Chen L, Gao D, Trush M, Wei C 2006 Increased mitochondrial reactive oxygen species production in newborn brain during hypoglycemia. Neurosci Lett 399:111-114

20. Chang YC, Tzeng SF, Yu L, Huang AM, Lee HT, Huang CC, Ho CJ 2006 Early-life fluoxetine exposure reduced functional deficits after hypoxic-ischemia brain injury in rat pups. Neurobiol Dis 24:101-113

21. Chew LJ, Takanohashi A, Bell M 2006 Microglia and inflammation: impact on developmental brain injuries. Ment Retard Dev Disabil Res Rev 12:105-112

22. Ivacko JA, Sun R, Silverstein FS 1996 Hypoxic-ischemic brain injury induces an acute microglial reaction in perinatal rats. Pediatr Res 39:39-47

23. Bona E, Andersson AL, Blomgren K, Gilland E, Puka-Sundvall M, Gustafson K, Hagberg H 1999 Chemokine and inflammatory cell response to hypoxia-ischemia in immature rats. Pediatr Res 45:500-509

24. Fan LW, Lin S, Pang Y, Rhodes PG, Cai Z 2006 Minocycline attenuates hypoxiaischemia-induced neurological dysfunction and brain injury in the juvenile rat. Eur J Neurosci 24:341-350

25. Blomgren K, Hagberg H 2006 Free radicals, mitochondria, and hypoxia-ischemia in the developing brain. Free Radic Biol Med 40:388-397

26. Bilbo SD, Levkoff LH, Mahoney JH, Watkins LR, Rudy JW, Maier SF 2005 Neonatal infection induces memory impairments following an immune challenge in adulthood. Behav Neurosci 119:293-301

27. Galic MA, Riazi K, Heida JG, Mouihate A, Fournier NM, Spencer SJ, Kalynchuk LE, Teskey GC, Pittman QJ 2008 Postnatal inflammation increases seizure susceptibility in adult rats. J Neurosci 28:6904-6913

28. Harre EM, Galic MA, Mouihate A, Noorbakhsh F, Pittman QJ 2008 Neonatal inflammation produces selective behavioural deficits and alters $N$-methyl-D-aspartate receptor subunit mRNA in the adult rat brain. Eur J Neurosci 27:644-653

29. Zhu C, Xu F, Wang X, Shibata M, Uchiyama Y, Blomgren K, Hagberg H 2006 Different apoptotic mechanisms are activated in male and female brains after neonatal hypoxia-ischaemia. J Neurochem 96:1016-1027

30. Chen CH, Toung TJ, Hurn PD, Koehler RC, Bhardwaj A 2005 Ischemic neuroprotection with selective kappa-opioid receptor agonist is gender specific. Stroke 36:1557-1561

31. Dogan MD, Ataoglu H, Akarsu ES 2000 Effects of different serotypes of Escherichia coli lipopolysaccharides on body temperature in rats. Life Sci 67:2319-2329

32. Nedrebo T, Reed RK 2002 Different serotypes of endotoxin (lipopolysaccharide) cause different increases in albumin extravasation in rats. Shock 18:138-141

33. Eklind S, Hagberg H, Wang X, Savman K, Leverin AL, Hedtjarn M, Mallard C 2006 Effect of lipopolysaccharide on global gene expression in the immature rat brain. Pediatr Res 60:161-168 